University of Nebraska - Lincoln

DigitalCommons@University of Nebraska - Lincoln

2012

Pore scale analysis of the impact of mixing-induced reaction dependent viscosity variations

\author{
S.M. Davison \\ Sandia National Laboratories, Albuquerque, NM \\ H. Yoon \\ Sandia National Laboratories, Albuquerque, NM \\ M. J. Martinez \\ Sandia National Laboratories, Albuquerque, NM
}

Follow this and additional works at: https://digitalcommons.unl.edu/usdoepub

Part of the Bioresource and Agricultural Engineering Commons

Davison, S.M.; Yoon, H.; and Martinez, M. J., "Pore scale analysis of the impact of mixing-induced reaction dependent viscosity variations" (2012). US Department of Energy Publications. 110.

https://digitalcommons.unl.edu/usdoepub/110

This Article is brought to you for free and open access by the U.S. Department of Energy at DigitalCommons@University of Nebraska - Lincoln. It has been accepted for inclusion in US Department of Energy Publications by an authorized administrator of DigitalCommons@University of Nebraska - Lincoln. 


\title{
Pore scale analysis of the impact of mixing-induced reaction dependent viscosity variations
}

\author{
S.M. Davison ${ }^{\mathrm{a}}$, H. Yoon ${ }^{\mathrm{b}}$, M.J. Martinez ${ }^{\mathrm{c}, *}$ \\ ${ }^{a}$ Chemical and Biological Systems, Sandia National Laboratories, P.O. Box 5800, MS-0734, Albuquerque, NM 87185-0734, United States \\ ${ }^{b}$ Geomechanics, Sandia National Laboratories, P.O. Box 5800, MS-0751, Albuquerque, NM 87185-0751, United States \\ ${ }^{\mathrm{c}}$ Thermal and Fluid Processes, Sandia National Laboratories, P.O. Box 5800, MS-0836, Albuquerque, NM 87185-0836, United States
}

\section{A R T I C L E I N F O}

\section{Article history:}

Received 14 September 2011

Received in revised form 21 December 2011

Accepted 28 December 2011

Available online 5 January 2012

\section{Keywords:}

Reactive transport

Transverse mixing

Transverse dispersion

Viscous thinning/thickening

Pore scale

\begin{abstract}
A B S T R A C T
Expanding interest in enhanced subsurface natural resource recovery and carbon sequestration motivates study of reacting flows in porous media. In this work, we examine the case of reaction products that increase or decrease the viscosity of the fluid. Parallel reactant streams flow through porous media and react transversely along the centerline. We utilize a pore scale, finite element numerical method that couples the reaction with fluid flow through two arrangements of porous media at three Damkohler $(D a)$ numbers and two viscosity conditions. When the product increases the fluid viscosity, the flow velocity is reduced and higher amounts of product are formed due to increased diffusion time. Conversely, reduced fluid viscosity leads to greater fluid velocity and lower amount of product formation. An exception is the viscous thinning case of high Peclet $(P e)$ number and high $D a$ where an instability develops (in low Reynolds $(R e)$ number flow) that enhances mixing between the reactants, resulting in increasing product formation.
\end{abstract}

(C) 2012 Elsevier Ltd. All rights reserved.

\section{Introduction}

The extent of mixing of reactive substances under flow conditions plays an important role in many natural and engineered systems including degradation of contaminants through chemical and biological processes [1,2], geochemical reactions during geological storage of $\mathrm{CO}_{2}[3,4]$, viscous fingering due to chemical reactions during petroleum recovery [5] and polymerization fronts [6]. Several works [7-9] have studied the effects of mixing on biochemical reactions, focusing on effects of transverse mixing between injected plumes (e.g. contaminants) and in situ groundwater. Another example of mixing-induced chemical reaction is related to the change of fluid properties such as the surface tension of immiscible interfaces or the fluid viscosities. The latter is, in particular, related to viscous fingering when viscosity is affected by chemically reactive solutes at the traveling front $[10,11]$. Viscous fingering induced by chemical reaction can also depend on the extent of mixing in the system where chemical reaction can increase or decrease the viscosity at the interface of two plumes. The change of fluid properties due to chemical reaction has the potential to motivate development of engineered systems for mixing-related environmental management.

There is extensive literature that details mechanisms of viscous fingering and fluid displacements in nonreactive systems $[5,12,13]$.

\footnotetext{
* Corresponding author. Tel.: +1 5058448729.

E-mail address: mjmarti@sandia.gov (M.J. Martinez).
}

In general, viscous fingering occurs when there is a displacement of a viscous fluid by a less viscous one. Viscous fingering due to immiscible displacement in porous media has also been examined $[14,15]$. In particular, flow instabilities during immiscible displacement after injection of $\mathrm{CO}_{2}$ into a saline aquifer were studied to account for the effects of viscosity ratio and permeabilities on the instability [16] and to investigate the effects of viscous, capillary, and gravitational forces on $\mathrm{CO}_{2}$ trapping mechanisms in homogeneous porous medium at pore scale [17]. In reactive systems, De Wit and Homsy $[10,11]$ studied viscous fingering in reactiondiffusion systems. More recently, Nagatsu and coworkers [18-20] have experimentally investigated miscible viscous fingering involving viscosity changes induced by chemical reactions over a range of the Damkohler number (i.e., the ratio of a characteristic time between fluid motion and chemical reaction) in a Hele-Shaw cell. Gérard and De Wit [21] numerically investigated the effect of a chemical reaction on viscous fingering dynamics in a system where the chemical product formed by mixing at the interface is more viscous than both replacing and displaced fluids. However, all these attempts are either performed in the absence of porous media, or based on continuum scale, and do not link mixing efficiency to the extent of mixing at pore scale which may play a significant role in chemical reaction in porous media.

A number of studies have indicated that continuum-based reactive transport models may overpredict the extent of mixing of substrates in porous media when pore-scale mixing and/or detailed features of heterogeneous patterns are not adequately 
characterized $[1,22,23]$. In the pore-scale investigations $[9,23,24]$, mixing-induced reactions occurred at pore scale to a much lesser extent, compared to a volume averaging scale for the continuumbased models, and the extent of mixing-induced reaction is strongly affected by porous media structure [9]. A key question is to determine values of transverse dispersivity that properly represent transverse mixing and reaction at the pore scale. Recently, Willingham et al. [25] demonstrated that a continuumscale model with accurate hydraulic conductivity values for two different pore distribution zones and one value of transverse dispersivity accurately matched total production formation by transverse mixing from a pore-scale model at a low Peclet number. The range of transverse dispersion coefficients in homogeneous, random, and flow focusing porous media were similar for both nonreactive and reactive species $[8,25]$. However, it is not clear whether this approach will be valid for reactive transport with viscosity variations due to chemical reaction. Tartakovsky [24] recently demonstrated that the difference between pore-scale and continuum scale modeling results increases with increasing Peclet number and Tartakovsky et al. [23] considered the density and viscosity of a fluid as a function of the solute concentration for a Rayleigh-Taylor instability. However, these attempts do not consider the change of fluid properties due to reaction. Our recently developed pore-scale model for reactive transport can be used to develop engineered fluids with adaptable properties (e.g., viscosity and density) for subsurface applications.

In this work, we present a new pore scale reactive transport model and use it to investigate the effect of reaction-induced viscosity variations on mixing and product formation in twodimensional (2-D) homogeneous porous media. The new pore scale reactive transport model was recently developed in a framework of coupled multiphysics simulation software for geosystems management and consists of a highly parallelized finite element analysis code for the Navier-Stokes equations coupled with mechanical, thermal, and chemical processes [26,27]. Model results are compared to an analytical solution for drag force around a circular cylinder for single phase flow [28] and published pore-scale simulation results for reactive transport with an instantaneous bimolecular reaction $[8,25]$. We apply the newly developed porescale model to evaluate transverse-mixing induced chemical reaction in porous media in contrast to the Hele-Shaw problems, resulting in a change in viscosity and velocity field as a function of product concentration. Transverse dispersion coefficients obtained from this work will be compared with cases without viscosity change due to chemical reaction in order to account for the effect of thinning and thickening viscosities on reaction.

The paper is organized as follows. In Section 2, we introduce the Navier-Stokes equations, reactive transport model for a simple $\mathrm{A}+\mathrm{B} \rightarrow \mathrm{C}$ reaction with and without viscosity modifications, and two configurations of porous media pattern used in this study. In Section 3, we verify the flow solution against an asymptotic analytical solution and demonstrate the results of two benchmarking studies involving transverse-mixing induced reaction in a uniform flow field without varing viscosity. In Section 4 , the results of thickening and thinning viscosity cases due to chemical reaction are presented before conclusions are presented in Section 5 where practical implications of this work are also discussed.

\section{Model system}

\subsection{Flow and transport equations}

Our model porous medium is composed of a regular array of cylinders with a uniform diameter (Fig. 1). Solutions of reactants $A$ and $B$ are separately introduced at the same mass flux over the upper and lower halves of the left boundary. Inlet concentrations for reactants $\mathrm{A}$ and $\mathrm{B}$ are $C_{A, 0}$ and $C_{B, 0}$, respectively. The reactant concentrations are assumed dilute and do not alter the solution density $(\rho)$ nor viscosity $(\mu)$ without chemical reaction. The pressure is held fixed over the right boundary. The bimolecular chemical reaction

$A+B \rightarrow C$

takes place at the interface where the two reactants meet. As a result, a product $C$ is formed, which alters the viscosity of the solution. The solution viscosity may increase or decrease with increasing product concentration. This chemically reactive transport problem can be described by the incompressible Navier-Stokes and species transport equations:

$$
\begin{aligned}
& \nabla \cdot \mathbf{u}=0 \\
& \rho \frac{\partial \mathbf{u}}{\partial t}+\rho \mathbf{u} \cdot \nabla \mathbf{u}=-\nabla p+\nabla \cdot[\mu(\nabla \mathbf{u} \\
& \left(\frac{\partial}{\partial t}+\mathbf{u} \cdot \nabla\right) C_{A}=D_{A} \nabla^{2} C_{A}-k C_{A} C_{B} \\
& \left(\frac{\partial}{\partial t}+\mathbf{u} \cdot \nabla\right) C_{B}=D_{B} \nabla^{2} C_{B}-k C_{A} C_{B} \\
& \left(\frac{\partial}{\partial t}+\mathbf{u} \cdot \nabla\right) C_{C}=D_{C} \nabla^{2} C_{C}+k C_{A} C_{B}
\end{aligned}
$$$$
\rho \frac{\partial \mathbf{u}}{\partial t}+\rho \mathbf{u} \cdot \nabla \mathbf{u}=-\nabla p+\nabla \cdot\left[\mu\left(\nabla \mathbf{u}+(\nabla \mathbf{u})^{T}\right)\right]
$$

where $\mathbf{u}$ is the velocity vector, $p$ is pressure, $C_{i}$ are concentrations with diffusion coefficients $D_{i}$ for species $\mathrm{A}, \mathrm{B}$, and C, and $k$ is the reaction rate constant. Eqs. (2) and (3) form the standard Navier-Stokes system, here written in terms of the full rate-of-deformation tensor, owing to the concentration-dependent viscosity. This dependence couples the flow system to the transport of reactant and product species, Eq. (4). In this work we will only consider inertia-free flows, however, the time dependent part of the acceleration term is retained because of the concentration dependence of the viscosity. This will be justified in the scaling of the equations below. If the viscosity is independent of the species concentrations, the flow problem is steady and uncoupled from the species transport. In the species transport equations, the diffusion is modeled by a simple Fickian form, with the possibility of different diffusion coefficients for each species, though here we will assume constant diffusion coefficients for all species. The source terms describe the mass rates of reaction, with rate constant $k$, from the bimolecular reaction described above.

In this work we consider a simple linear model of concentrationdependent viscosity of the form

$\mu=\mu_{0}\left(1+\mu^{\prime} C_{C}\right) ; \quad \mu^{\prime}=\frac{1}{\mu_{0}} \frac{d \mu}{d C_{C}}$.

where $\mu_{0}$ is the viscosity of the solvent. We consider both thickening $\left(\mu^{\prime}>0\right)$ and thinning $\left(\mu^{\prime}<0\right)$ viscosity with product concentration. In cases where the viscosity is independent of product concentration, researchers have studied how the mechanical dispersion in this model porous medium is altered by this reactive system. Here we study the interplay of reaction-induced viscosity variations on dispersion and on stability of the flow. Thinning viscosity with concentration in displacement problems is known to promote viscous fingering [5,21].

We scale the governing equations to reveal the important terms and their association with key physical mechanisms. In the equations to follow, lengths are nondimensionalized with $l$, the spacing between cylinders, velocity with $U$, the average velocity of the injected solution, pressure and stress with $\mu_{0} U / l$, and time with $\tau_{D}=l^{2} / D_{C}$, the diffusion time scale for the product. Concentrations are nondimensionalized with $C_{A, 0}$, the injected value of reactant A concentration. This introduces $C_{B, 0} / C_{A, 0}$ as a system parameter, 


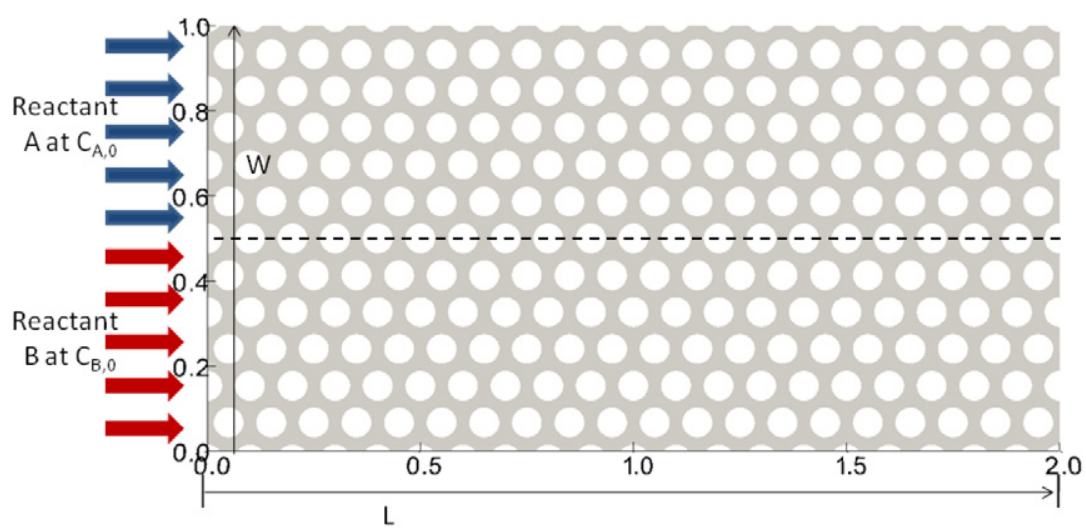

(a)

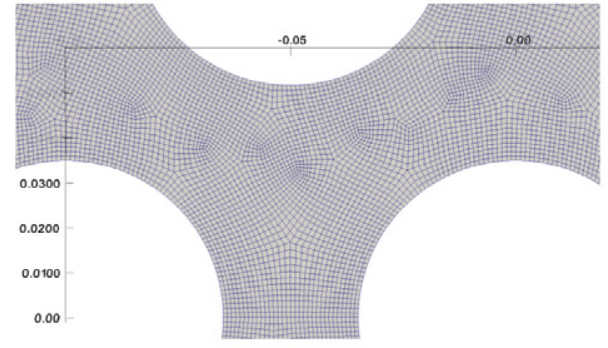

(b)

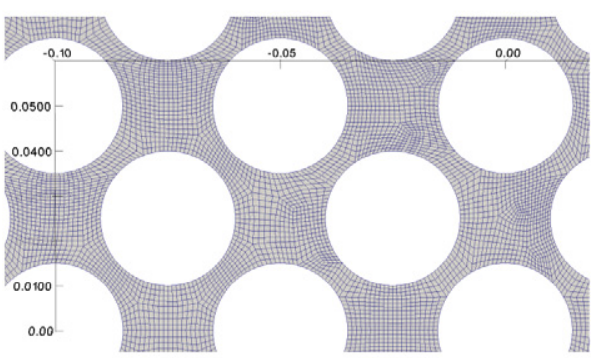

(c)

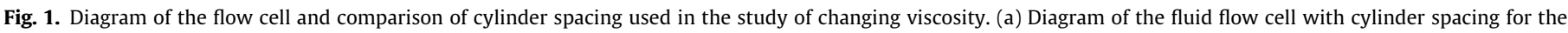
loosely packed array. (b) Cylinder arrangement detail for loosely packed array. (c) Cylinder arrangement for closely packed array.

but we will assume this is unity here. We also introduce, for generality, the ratios of reactant to product diffusion coefficient ratios, $\delta_{A}=D_{A} / D_{C}$ and $\delta_{B}=D_{B} / D_{C}$. In the nondimensional equations below, we will retain the same symbols as in the dimensional equations; the context of the discussion should make the distinction clear. With this scaling, the continuity Eq. (2) remains unchanged, while the remaining equations become:

$S c^{-1} \frac{\partial \mathbf{u}}{\partial t}+\operatorname{Reu} \cdot \nabla \mathbf{u}=-\nabla p+\nabla \cdot\left[\bar{\mu}\left(\nabla \mathbf{u}+(\nabla \mathbf{u})^{T}\right)\right]$

$\left(\frac{\partial}{\partial t}+P e \mathbf{u} \cdot \nabla\right) c_{A}=\delta_{A} \nabla^{2} c_{A}-D a c_{A} c_{B}$

$\left(\frac{\partial}{\partial t}+\operatorname{Peu} \cdot \nabla\right) c_{B}=\delta_{B} \nabla^{2} c_{B}-D a c_{A} c_{B}$

$\left(\frac{\partial}{\partial t}+\operatorname{Peu} \cdot \nabla\right) c_{C}=\nabla^{2} c_{C}+\operatorname{Da} c_{A} c_{B}$

where $c_{i}$ is the dimensionless concentration of species $i$. In this work we assume creeping flow conditions, $R e=\rho U l / \mu_{0} \rightarrow 0$, so we are left with the Schmidt, Peclet and Damkohler numbers as our dimensionless parameters:

$S c=\frac{\mu_{0}}{\rho D_{C}} \quad P e=\frac{U l}{D_{C}} \quad D a=\frac{k c_{A, 0} l^{2}}{D_{C}}$.

The Schmidt number, which measures the timescale for vorticity diffusion compared to molecular diffusion, appears in the momentum equations, rather than the species transport equations, because we have scaled by the species diffusion time; this choice is motivated by the fact that the transverse reaction problem is diffusion-limited. In these equations, the Damkohler number measures the diffusion to reaction time ratio: $D a=\tau_{D} / \tau_{r x n}$, where the reaction time is $\tau_{r x n}=1 / k c_{A, 0}$. This scaling also introduces the dimensionless concentration-dependent viscosity function as, $\bar{\mu}=\mu / \mu_{0}=1+\bar{\mu}^{\prime} c_{C} \quad \bar{\mu}^{\prime}=\frac{C_{A, 0}}{\mu_{0}} \frac{d \mu}{d C_{C}}$

in which $\bar{\mu}^{\prime}$ is the dimensionless fractional change in viscosity.

Notice that summing either of the reactant transport equations with the product equation, assuming uniform diffusion coefficients, shows that the summed concentration is conserved. This property has been exploited in previous work $[8,29]$, to derive an asymptotic expression for the concentrations under instantaneous reaction conditions. This expression, discussed below, forms the basis for analysis of the hydrodynamic dispersion under reactive transport conditions.

\subsection{Numerical method}

Owing to the general geometries encountered in pore scale models of porous materials, unstructured grid methods are preferred to accommodate complex geometry. In this work we use finite element methods to solve the coupled incompressible fluid dynamics and species transport equations. Equal-order basis functions for velocity-pressure pairs are preferred, allowing simple and uniform data structures, but mainly to facilitate solution of large algebraic problems resulting from high resolution grids necessary to capture complex geometry. To overcome the fact that equalorder velocity-pressure pairs violate the LadyshenskayaBabuska-Brezzi (LBB) [30] stability condition, low-order pairs are usually supplemented by stabilization procedures to remove spurious pressure modes. In this work we apply the polynomial pressure projection stabilization (PPPS) method introduced by Dohrmann and Bochev [31] and Bochev et al. [32] for the Stokes flow problem, and later shown to also apply to the Navier-Stokes equations [33].

The weak form of the PPPS formulation [33] for the NavierStokes system is, using standard notation, 


$$
\begin{gathered}
\int_{\Omega} \mathbf{w} \cdot\left(\rho \frac{\partial \mathbf{u}}{\partial t}+\rho \mathbf{u} \cdot \nabla \mathbf{u}\right) d \Omega+\int_{\Omega} \nabla \mathbf{w}: \tau d \Omega-\int_{\Gamma} \mathbf{w} \cdot \tau \cdot \mathbf{n} d \Gamma \\
\quad+\int_{\Omega} q \nabla \cdot \mathbf{u} d \Omega-\int_{\Omega} \frac{1}{\mu}(p-\pi p)(q-\pi q) d \Omega=0
\end{gathered}
$$

with stress tensor defined by

$\boldsymbol{\tau}=-p \mathbf{I}+\mu\left(\nabla \mathbf{u}+(\nabla \mathbf{u})^{T}\right)$.

The first four terms in the weak formulation would form the standard Galerkin finite element method, in which $\mathbf{w}$ and q would be an LBBconforming velocity-pressure weight function set, of unequal order. The last term is the PPPS stabilizing term which provides the stabilization needed for allowing equal-order velocity-pressure basis functions. When finite element approximations are employed, the projection operator in the stabilizing term, $\mathbf{A}_{\pi} \mathbf{p}$, is obtained as the assembly of element matrices [31],

$\mathbf{A}_{\pi e}=\left(\mathbf{M}_{e}-\mathbf{E}_{e}^{T} \mathbf{D}_{e}^{-1} \mathbf{E}_{e}\right) / \mu_{e}$

where

$\mathbf{M}_{e}=\int_{\Omega_{e}} \mathbf{N N}^{T} d \Omega ; \quad \mathbf{E}_{e}=\int_{\Omega_{e}} \mathbf{a}(\mathbf{x}) \mathbf{N}^{T} d \Omega ; \quad \mathbf{D}_{e}=\int_{\Omega_{e}} \mathbf{a} \mathbf{a}^{T} d \Omega$.

In these equations $\mu_{e}$ is viscosity in element $e, \mathbf{N}$ is a vector of standard pressure basis functions for the element, and $\mathbf{a}(\mathbf{x})$ is a vector of low-order polynomials of the spatial coordinates. Details and analysis of the method are given in Dohrmann and Bochev [31] and Bochev et al. [32]. An attractive feature of this scheme is that there are no adjustable parameters or scalings in the stabilization term. In the present application, this stabilization allows the use of equal-order $Q_{1} Q_{1}$ velocity-pressure pairs, and $Q_{1}$ for the species concentrations. The PPPS method is implemented in the parallel-processing Sierra/ Aria flow code for a variety of two and three-dimensional finite elements [27]. The stabilization allows solutions of large algebraic systems on massively parallel-processing computers using standard linear solvers (e.g., ilu/gmres in our application) implemented for parallel computers. The finite element discretization method was also used to solve the advection-diffusion equations. This method is second-order accurate $[34,35]$. To eliminate deleterious effects of numerical diffusion, grid refinement studies were conducted to ensure the solutions presented later are grid-independent.

\subsection{Model problems}

Fig. 1 shows a two-dimensional computational domain of length $\mathrm{L}(2 \mathrm{~cm})$ and width $\mathrm{W}(1 \mathrm{~cm})$, containing an array of solid cylinders. Initially, the pore space is saturated with solution without reactants. On the inflow boundary on the left, the dilute solutions of reactants are introduced at uniform and constant mass flux rate, $\rho U$. Solution with reactant $\mathrm{A}$ is introduced over the upper half of the boundary and solution with reactant B over the lower half of the boundary. For the species transport this means,

$$
\begin{aligned}
& C_{A}(t=0)=C_{B}(t=0)=C_{C}(t=0)=0 \\
& C_{A}(x=0, t>0)= \begin{cases}0 & \text { for } 0<y<W / 2 \\
C_{A, 0}=C_{0} & \text { for } W / 2<y<W\end{cases} \\
& C_{B}(x=0, t>0)= \begin{cases}C_{B, 0}=C_{0} & \text { for } 0<y<W / 2 \\
0 & \text { for } W / 2<y<W\end{cases} \\
& C_{C}(x=0, t>0)=0 \text { for } y \geqslant 0
\end{aligned}
$$

The right side is an outflow boundary, on which a uniform pressure is specified, and over which the species are allowed to freely exit the boundary. The upper and lower boundaries are no-flow boundaries for species, and no-slip on the solid cylinder portion for flow. In the interior, along each cylinder the no-slip and zero species flux are specified:

$$
\begin{aligned}
& \mathbf{u}=0, \quad \mathbf{x} \in \Gamma_{s} \\
& -D_{i} \nabla C \cdot \mathbf{n}=0, \quad \mathbf{x} \in \Gamma_{s}
\end{aligned}
$$

where $\mathbf{n}$ is the outward-pointing unit normal vector to the boundaries of the cylinders, denoted collectively by $\Gamma_{s}$.

Two different arrays of cylinders are used to compare the effect of porous medium structure on reactive transport. Fig. 1 compares the two arrays considered in this study, including an example of the grids used. The "loosely packed" array is a hexagonal packing, with diameter $(d)=0.07 \mathrm{~cm}$ and the closest center-to-center distance between two cylinders $(l)=0.1 \mathrm{~cm}$, and porosity $(\phi)=0.556$, while the "closely packed" array is a square packing (rotated by 45 degrees to the average flow direction), with $d=0.03 \mathrm{~cm}, l=0.05 / \sqrt{2} \mathrm{~cm}$, and $\phi=0.434$.

For all simulations, the pore structures and fluids were specified to mimic pore-scale experiment and numerical simulations for reactive transport induced by transverse mixing $[9,25]$. Density $(\rho)$ and viscosity $\left(\mu_{0}\right)$ for aqueous solutions are specified as $1 \mathrm{gm} /$ $\mathrm{cm}^{3}$ and $0.01 \mathrm{gm} / \mathrm{cm} / \mathrm{s}$, respectively. The diffusivity $\left(D_{i}\right)$ of all species in the solution is $10^{-5} \mathrm{~cm}^{2} / \mathrm{s}$. The average velocity $(U)$ of solution through pore space along the inlet boundary is $0.02 \mathrm{~cm} / \mathrm{s}$, such that $R e<1$ for all cases. Inlet concentrations $\left(C_{0}\right)$ for both reactants are 0.5 in all problems considered in this work. For mixing-induced reaction problems, it has been demonstrated $[24,25]$ that the diffusive and mechanical dispersion processes that control the degree of mixing in 2-D porous media also affect mixing in 3-D porous media. Although our 2-D numerical simulations do not capture all of the physics present in real 3D porous media (e.g., twisting of streamlines and different diffusion length scale), this work allows us to isolate and quantify these effects, particularly, associated with the change of viscosity due to chemical reaction.

\section{Numerical verification}

\subsection{Drag force on an array of cylinders}

The flow solver is verified by computing the drag on a typical cylinder due to a steady non-reactive flow through a hexagonal array of cylinders. Fig. 2 compares the drag,

$F=\int_{\Gamma_{s}} \mathbf{n} \cdot \tau \cdot \mathbf{n} d \Gamma$

on an individual cylinder as a function of cylinder volume fraction $(1-\phi)$ between the present numerical scheme and the analysis of Sangani and Acrivos [28]. Many researchers have studied this problem and given the mapping of the cylinder drag to permeability to flow, and also compared to Carmen-Kozeny model [36,37]. The calculation was performed on three individual cylinders to verify a periodic solution on non-periodic, non-uniform grids. Fig. 2 shows the comparison is excellent over a wide range of porosities, over which the drag varies by three orders-of-magnitude.

\subsection{Reaction along a transverse mixing zone with uniform viscosity}

To verify the solution of the reactive transport, independent of the Navier-Stokes equations, we solve the reactive flow problem with boundary and initial conditions (Eq. (14)) in a uniform flow field (i.e., no vertical flow) in an open channel (i.e., the absence of cylinders). In the case of an instantaneous reaction (e.g., very high $k$ value in Eq. (4)), the reaction occurs along the centerline where species A and B mix due to molecular diffusion. For equal diffusion coefficient for all species, a continuum scale analytical solution for the product $\left(C_{c}\right)$ formed by the transverse mixing of two parallel streams is given by $[29,38]$ 


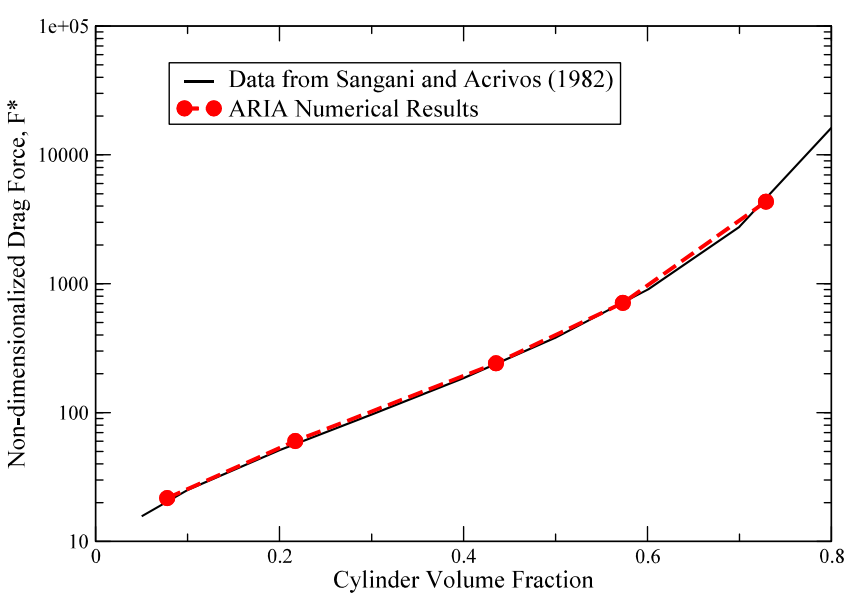

Fig. 2. Comparison of the non-dimensional drag force computed by the PPPSstabilized finite element method to results published by Sangani and Acrivos [28].

$C_{C}(x, y)=\frac{C_{0}}{2}\left[\operatorname{erfc}\left(\frac{y}{2 \sqrt{D(x / v)}}\right)\right]$

where $D$ is the molecular diffusion coefficient in an open channel or the transverse dispersion coefficient $\left(D_{T, R}\right)$ in porous media, $x$ is the distance from the inlet, $y$ is the distance from the centerline, and $v$ is the average linear velocity. It is noted that for transient diffusion $x / v$ in Eq. (18) represents time. Comparison of the effect of the value of reaction rate constant $(k)$ in the numerical solution to the analytic solution (Eq. (18)) is shown in Fig. 3. The numerical solution matches the product profile of Eq. (18) with $k=10$ within $8 \%$ at the peak concentration and within less than $1 \%$ in terms of total product mass integrated over the cross-flow direction $(y)$. Based on these results, a $k$ value of 10 was used as the maximum $k$ value for the reactive transport problems with viscosity variations in porous media in the next section.

We also verify the solution of the reactive transport in a porous medium in the case of uniform viscosity. One of the key parameters to represent transverse mixing and product formation is the transverse dispersion coefficient $[8,9,25]$. To obtain the transverse dispersion coefficient, the total product mass can be computed by

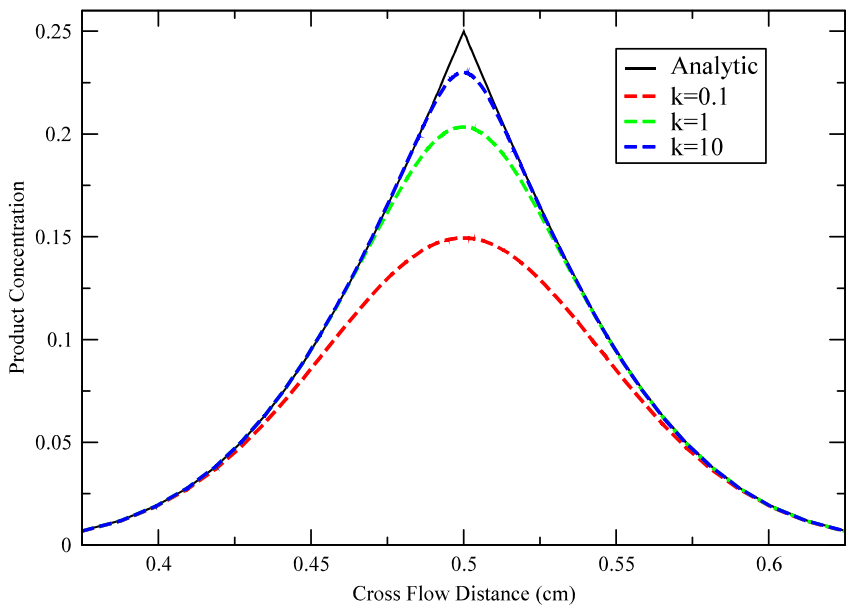

Fig. 3. Comparison of the effect of value of reaction constant, $k$, in the numerical solution to the analytic solution (Eq. (18)) assuming instantaneous reaction $(k \rightarrow \infty)$. Product concentration in the cross flow direction at the channel outflow boundary $(\mathrm{X}=\mathrm{L})$ is shown with distance measured from the bottom of the domain $(0.5 \mathrm{~cm}$ is on the centerline). integrating the product concentration (Eq. (18)) over the crosssection to the main flow direction and is given by

$m(x)=\int C_{c} d y=2 C_{0} \phi \sqrt{\frac{x D_{T, R}}{\pi v}}$

where $m(x)$ is the total mass of the product at the distance $\mathrm{x}$ from the inlet along the main flow direction $(x)$ and $D_{T, R}$ is the transverse dispersion coefficient based on the product profile. A simple way to obtain $D_{T, R}$ is to integrate the product mass (Eq. (19)) up to a given point, $X$, and $D_{T, R}$ is given by

$D_{T, R}=\frac{1}{X^{3}} \frac{9 \pi v}{16 C_{0}^{2} \phi^{2}}\left[\int_{0}^{X} m(x) d x\right]^{2}$

This approach was also used to accurately obtain $D_{T, R}$ for a variety of porous media distributions using the Lattice Boltzmann method for fluid flow and a finite volume method for reactive transport (LBFVM) $[8,9,25]$, which represents the extent of transverse-mixing limited reactions in flowing conditions. The results from the current finite element method are compared to those from the LBFVM implementation of Yoon et al. [39]. Fig. 4 compares the total product mass (Eq. (19)) along the main flow direction for two pore-scale modeling results with the analytical solution. The $D_{T, R}$ value for the analytical solution (Eq. (19)) was obtained using Eq. (20). The difference between numerical results for both methods and the analytical solution can be attributed to the effect of a finite value of $k$ (i.e., $k=100$ ) used in the numerical results; the LBFVM with fully instantaneous reaction matched the analytical solution accurately in Acharya et al. [8]. The difference between the two numerical methods can be attributed to the different size of mesh, shape of cylinders, and slightly different pore spacing due to a uniform grid spacing for Lattice Boltzmann method, but the difference was not significant. The preliminary results for other cases show that the ARIA results match the LBFVM results relatively well [26], giving confidence in the ability of the current formulation to model fluid flow with reactions at pore scale.

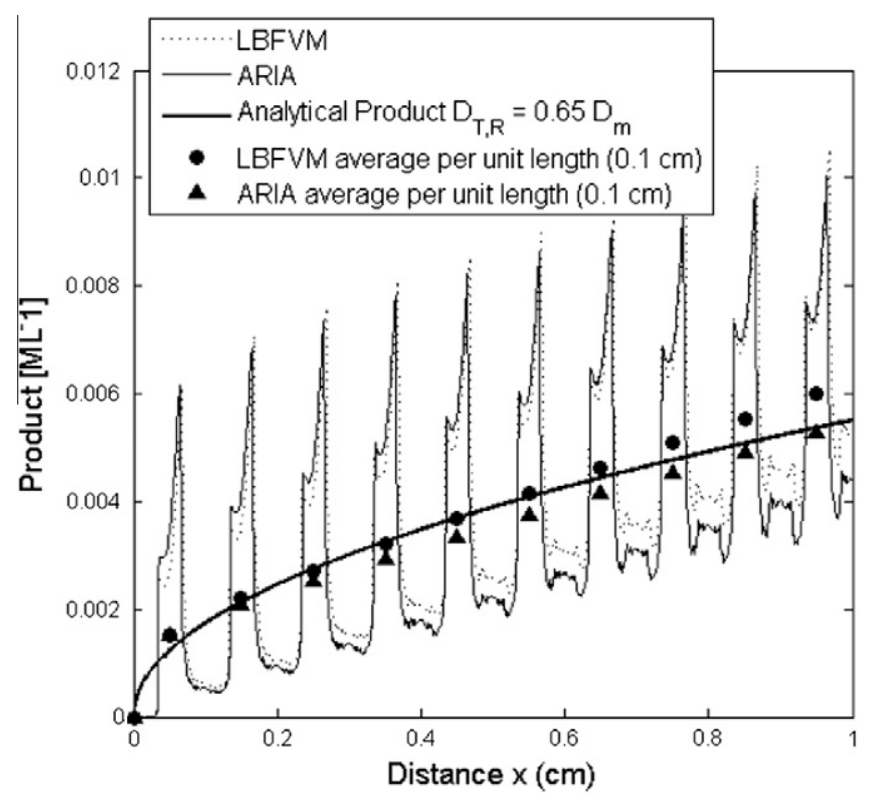

Fig. 4. Profiles of the total product mass as a function of distance from the inflow boundary along the main flow direction $(\mathrm{x})$ for the LBFVM and finite element (ARIA) method at a grid scale ( $\sim 10$ microns) and at an average scale per unit length $(0.1 \mathrm{~cm})$. The analytical solution (Eq. (19)) is also shown using $\mathrm{D}_{T, R}$. 

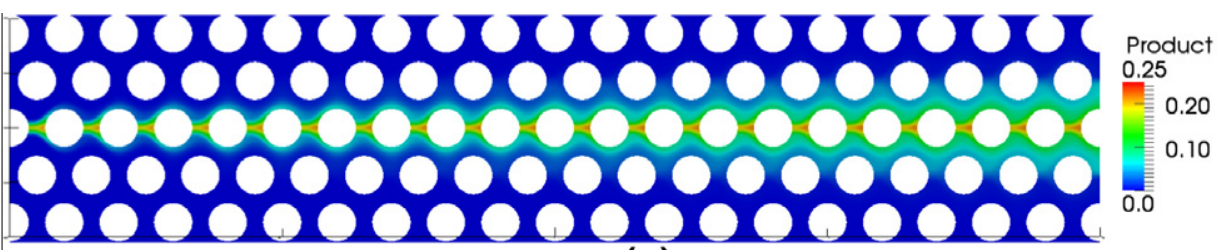

(a)
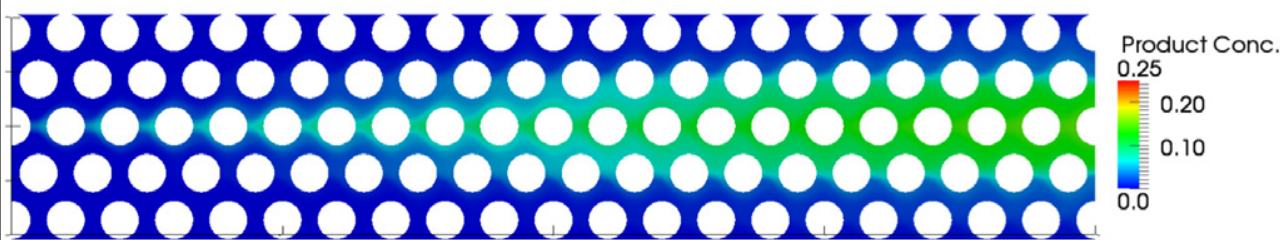

(b)

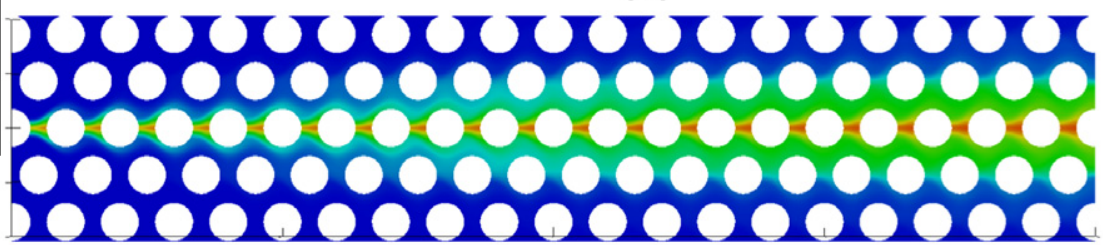

(c)

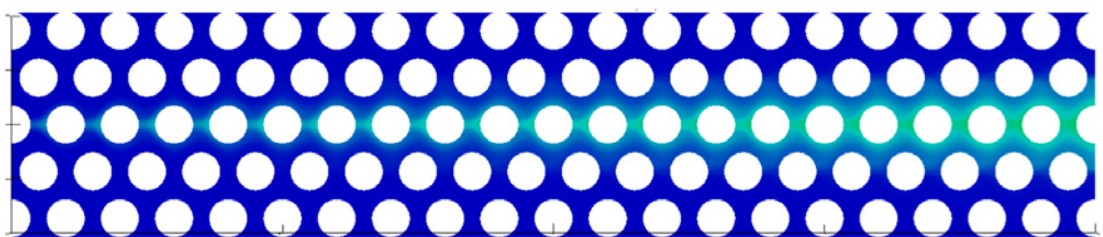

(d)

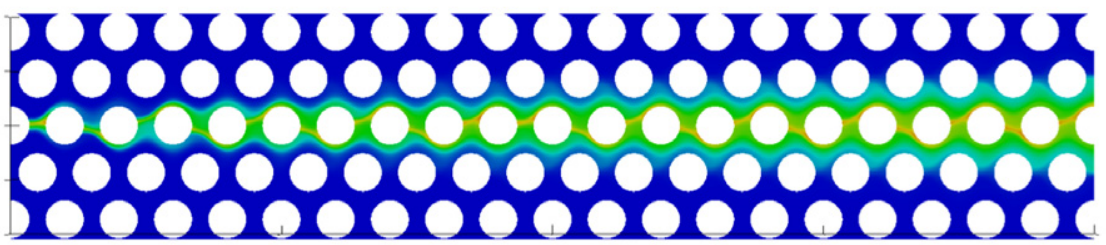

(e)

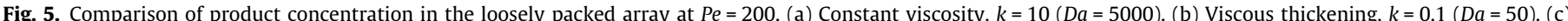

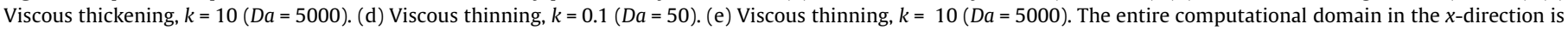
shown, while the $y$-direction is limited to $0.3<\mathrm{y}<0.7 \mathrm{~cm}$.

\section{Reaction along a transverse mixing zone with concentration- dependent viscosity}

\subsection{Effect of viscosity variations on the extent of mixing and product formation}

To evaluate the effect of concentration-dependent viscosity on reaction due to transverse mixing, we consider two behaviors of viscosity variation with product concentration, thinning and thickening. In both cases the viscosity increases or decreases by a factor of 10 from the influent viscosity due to chemical reaction. The theoretical maximum product concentration is half of influent concentration (i.e., 0.25) from Eq. (18). For the thinning and thickening cases, the value of viscosity changes linearly from $0.01 \mathrm{gm} / \mathrm{cm} / \mathrm{s}$ to 0.001 and $0.1 \mathrm{gm} / \mathrm{cm} / \mathrm{s}$, respectively, over the range of product concentration from 0.0 to 0.25 . This can be expressed in terms of the fractional change in viscosity:

$\frac{\Delta \mu}{\mu_{\text {avg }}}= \begin{cases}-1.636 & \text { thinning } \\ +1.636 & \text { thickening }\end{cases}$

where $\mu_{\text {avg }}$ is the average of the maximum and minimum viscosity over the range of product concentration. Notice that while the
Schmidt number in Eq. (8) is constant (i.e., $S c=1000$ ), the local Schmidt number, based on the local value of viscosity, varies by as much as 10 -fold over the domain. This range of viscosity variation is moderate compared to the reaction-induced viscosity variations in the experiments of Nagatsu and co-workers [18-20] and the shear-thinning variations reported in Zhong et al. [40].

Since the flow field is also affected by viscosity variations, in contrast to the constant viscosity case, the flow is coupled to the species transport and the system is solved as a transient problem, until a steady solution for both flow and concentration fields is determined. Steady solutions were found in all cases, except for one which displayed two admissible steady configurations as discussed below. Grid studies were conducted to identify and eliminate numerical errors. All results presented used the most refined grids which displayed grid-independence. For both thinning and thickening viscosity cases, three values of the reaction rate constant $(k)$ were considered $(k=0.1,1$, and 10$)$ for testing the effect of reaction rate on the extent of mixing. As discussed in Section 3.2, the maximum value of $k=10$ is a reasonable approximation to instantaneous reaction, while preserving computational efficiency.

Figs. 5 and 6 show steady distributions of the product concentration for the loosely and closely packed arrays, respectively, for 


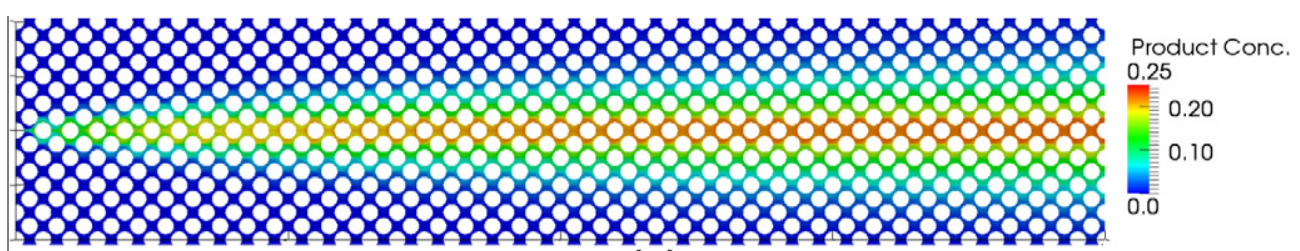

(a)

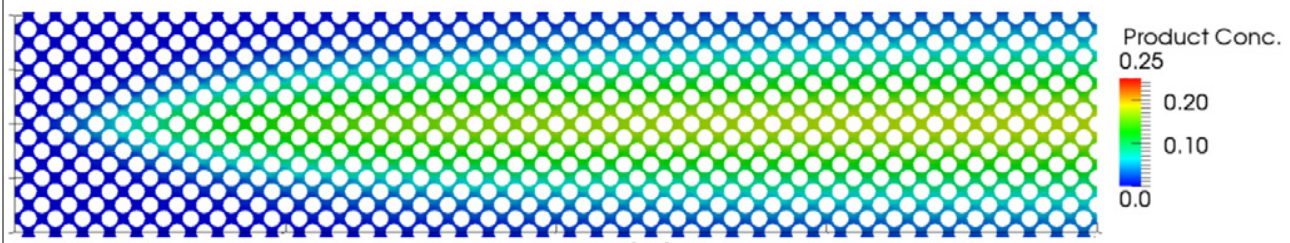

(b)

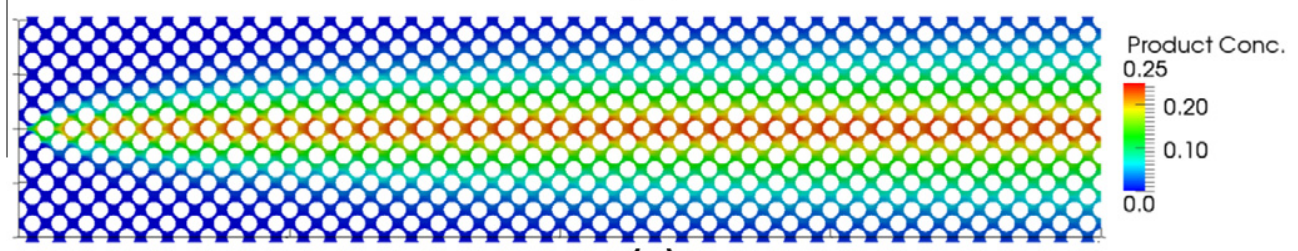

(c)

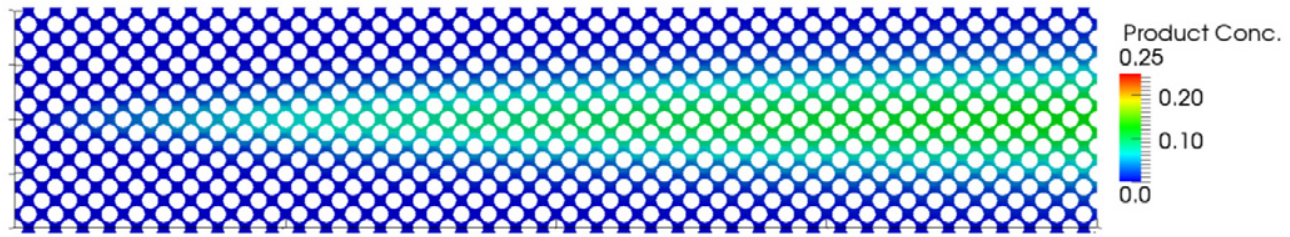

(d)

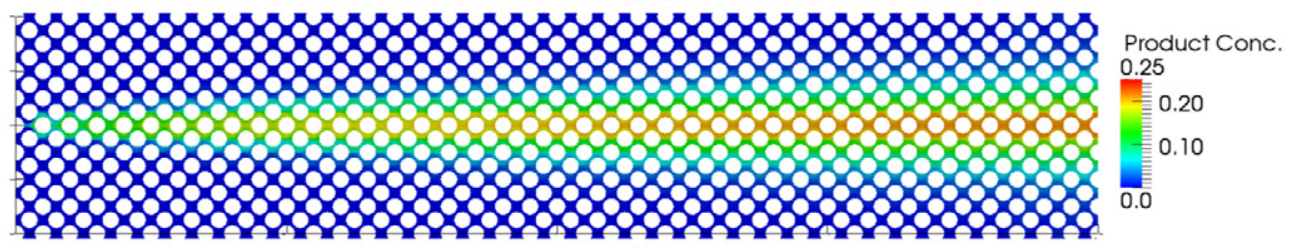

(e)

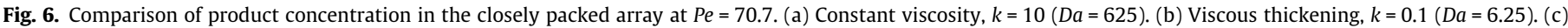

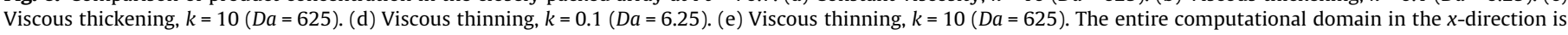
shown, while the $y$-direction is limited to $0.3<\mathrm{y}<0.7 \mathrm{~cm}$.

cases of constant viscosity (Figs. 5 and 6 (a)), thickening viscosity (Figs. 5 and 6 (b) and (c)), and thinning viscosity (Figs. 5 and 6 (d) and (e)). The effect of thinning vs. thickening viscosity on product formation is compared for the highest and lowest $k$ values, while only the highest $k$ value case is included for constant viscosity. Since each reactant is introduced separately over the upper or lower half of the inlet, the reaction commences at the centerline on the downstream side of the first (half) cylinder where reactants first meet. As the solution flows downstream past the array of cylinders, the reaction proceeds by transverse diffusion, resulting in spreading of the product plume along the main flow direction. For the same $k$ value in both arrays, the thickening viscosity increases the reaction zone in a transverse direction compared to the thinning viscosity (e.g. Fig. 5(b) vs. (d) or Fig. 6 (b) vs. (d)).

The effect of altering viscosity on the flow field is illustrated in Fig. 7. Fig. 7 shows the velocity field near the outflow boundary of the loosely $(\mathrm{a}-\mathrm{c})$ and closely $(\mathrm{d}-\mathrm{f})$ packed arrays for constant (a \& d), thickening (b \& e), and thinning (c \& f) viscosities. Thickening viscosity creates more resistance to flow where product is formed, resulting in spreading of the flow field around the region of higher viscosity. Velocity along the centerline is reduced due to higher viscosity, thereby longer diffusional time exists within the region of higher viscosity and more product formation results. Conversely, thinning viscosity reduces flow resistance and results in "focusing or channeling" of the product, an effect reminiscent of flow focusing pore structure [9] or viscous fingering in miscible systems due to chemical reaction $[10,11]$. The region of higher velocity near the centerline suppresses transverse mixing, resulting in less product formation. Also, note the effect of packing geometry and porosity at constant viscosity. The loosely packed hexagonal array shows high-speed predominantly horizontal pathways, with large stagnation regions between cylinders, while the closely packed array displays a periodic pattern of high speed flow through pore throats (i.e. the minimum gap between cylinders), with slower flow everywhere else.

The channeling effect in viscous thinning cases is further exacerbated by the Peclet number, here illustrated by comparing product solutions on the two arrays. Though they have similar porosities, 0.556 for the loosely packed versus 0.434 for the closely packed, the pore throat gap spacing is quite different, $0.03 \mathrm{~cm}$ compared to $0.00535 \mathrm{~cm}$, respectively. The dimensionless gap spacing $((l-d) / l)$ is a factor of two different, 0.3 in the loosely packed array 


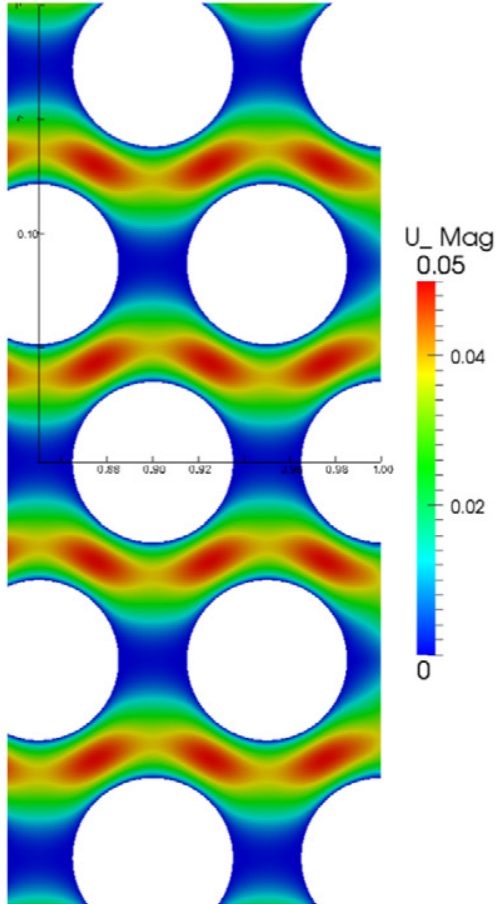

(a)

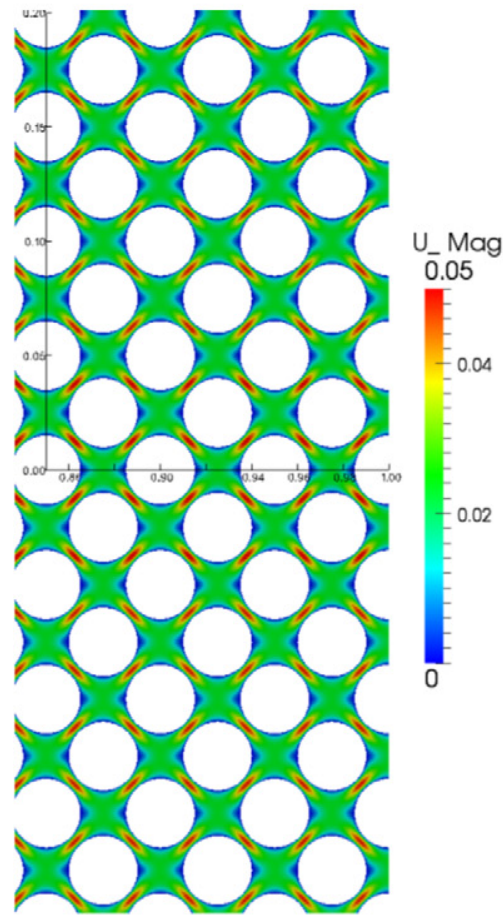

(d)

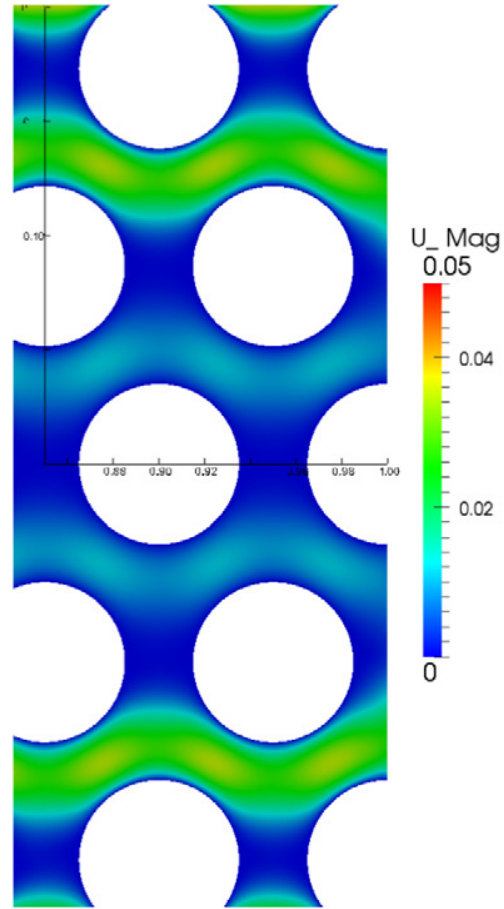

(b)

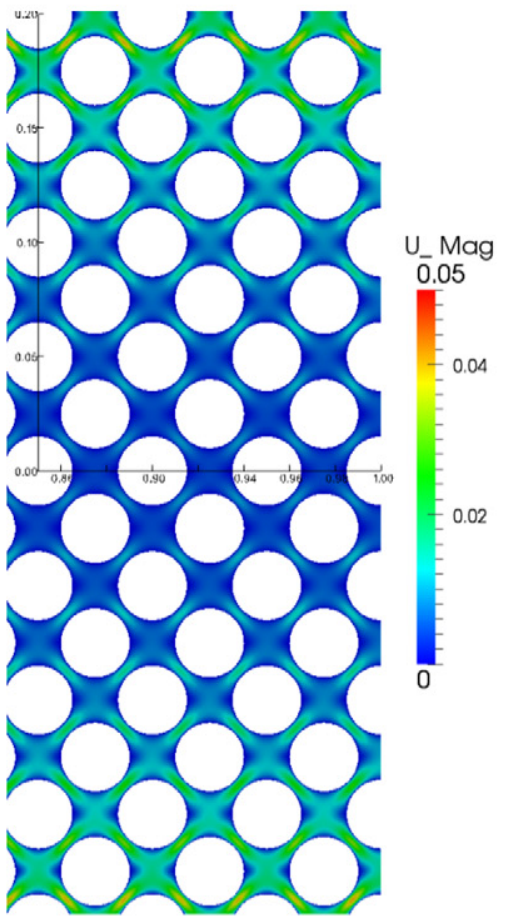

(e)

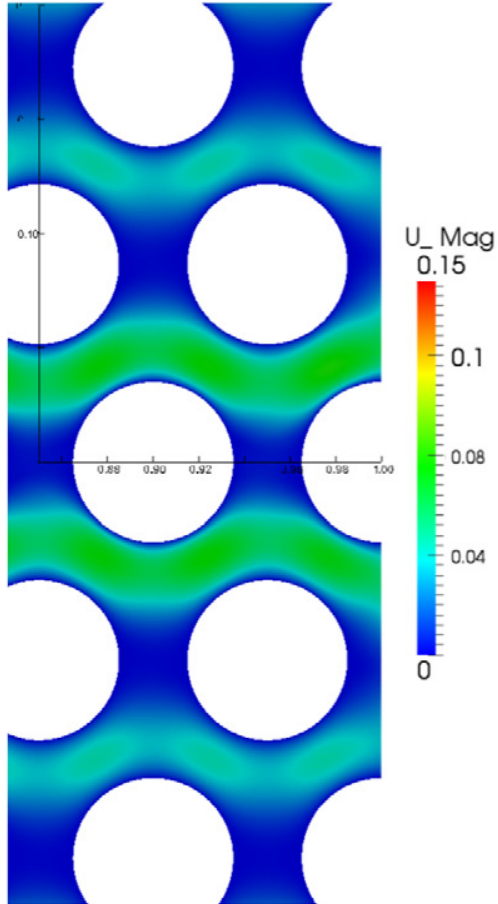

(c)

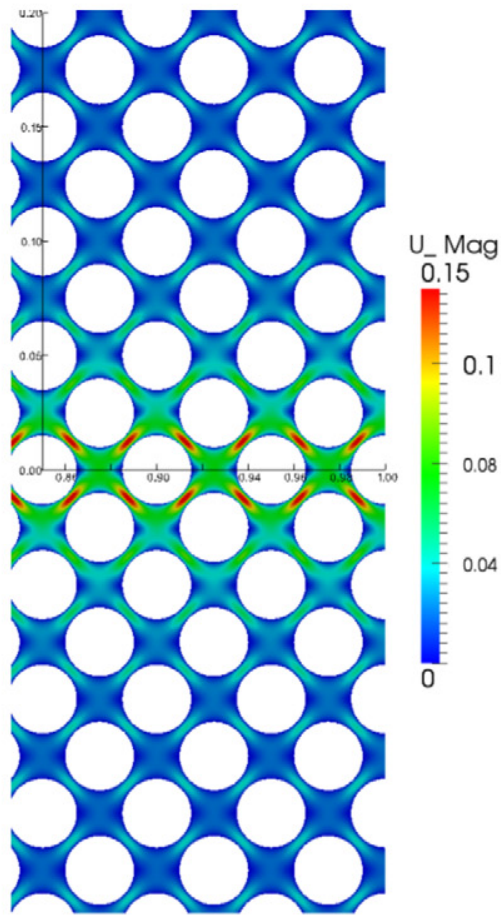

(f)

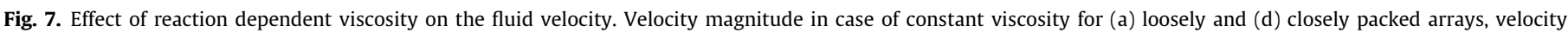

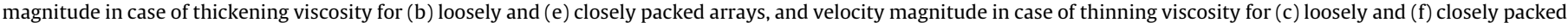
arrays. Note change in colorbar range.

compared to 0.1515 in the closely packed array. Flow resistance increases as the pore throat decreases. The solution in Fig. 5 (e) for high Peclet and Damkohler number, and for viscous thinning, shows a very thin band of high concentration of product (therefore a thin band of reaction zone) which alternately passes over the top and bottom of successive cylinders. This illustrates the grid resolution needed for this parameter set to resolve this extremely thin reaction zone. This figure is not the steady solution, but shows a time value where the noted sinuous reaction zone is propagating downstream in a wave-like pattern, switching from one configuration to another at a particular point in space. The final "steady" solution was found (not shown, but very similar to this one) by successively halving the mesh spacing until a stationary pattern was found. This required a resolution of 750,000 grid points on 


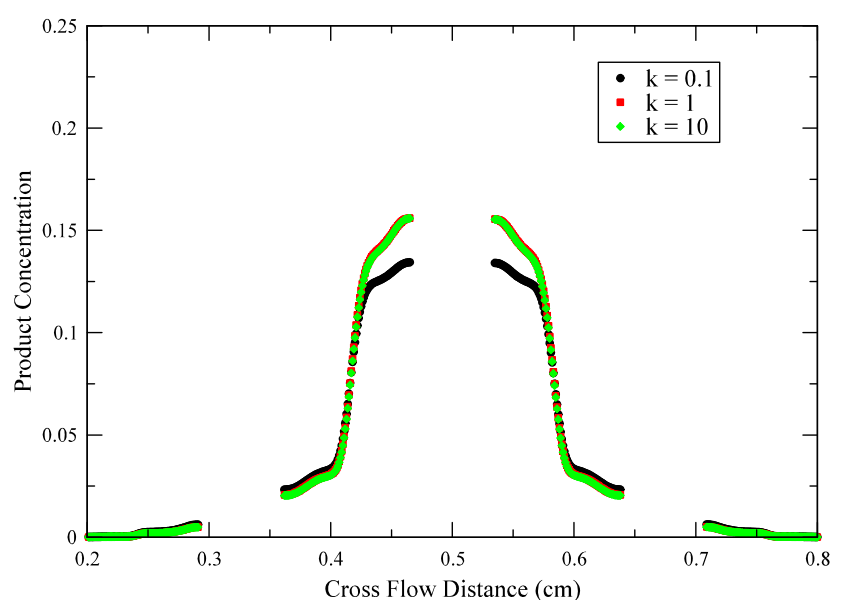

(a)

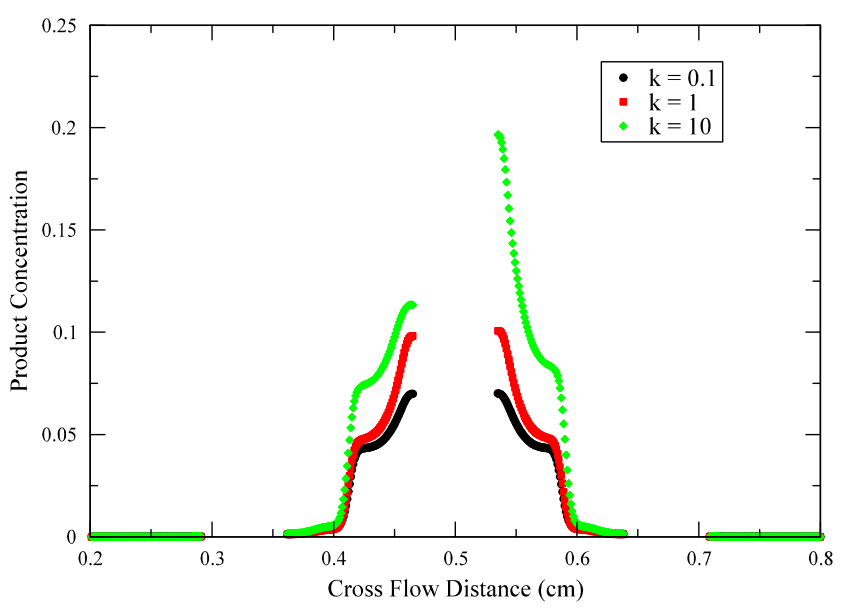

(b)

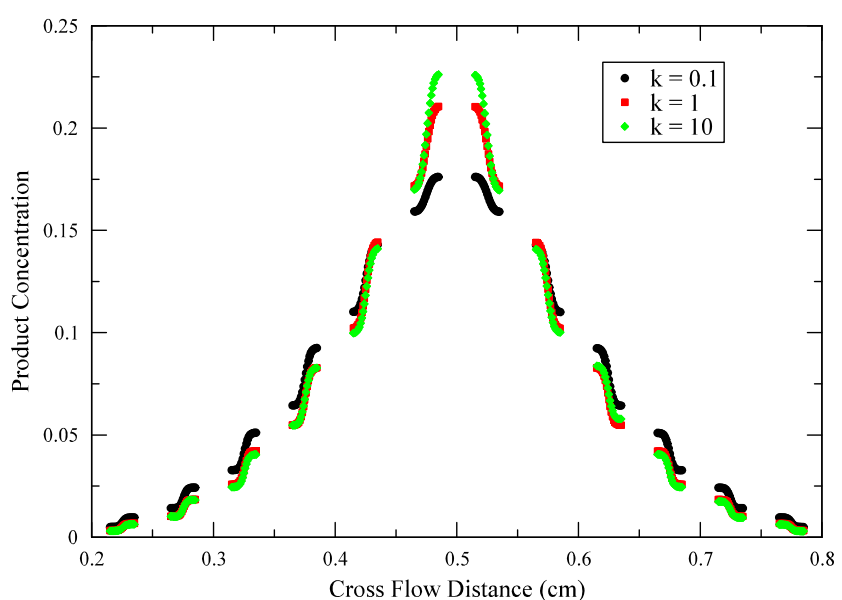

(c)

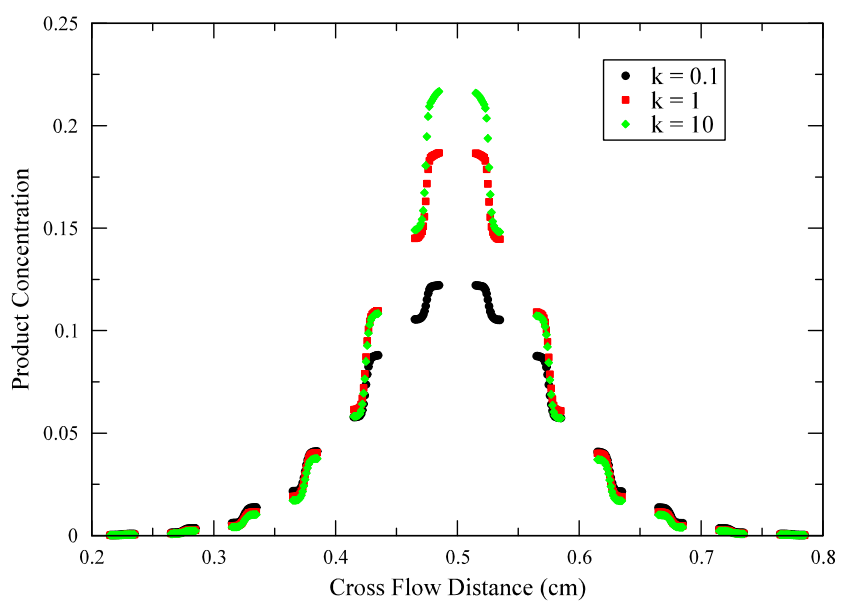

(d)

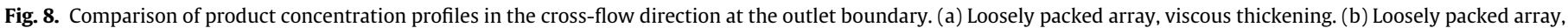
viscous thinning. (c) Closely packed array, viscous thickening. (d) Closely packed array, viscous thinning.

which two conditionally stable patterns were computed, one was the odd solution of the other with respect to the axial centerline. This may well suggest further instabilities for larger Pe and $D a$.

For the same $k$ value and viscosity type, the width of reaction zone is wider in the closely packed array than the loosely packed array (Figs. 5 and 6). In Willingham et al. [9], for both small and large cylinder diameter arrays with the same porosity, product formation was nearly identical. It was concluded in Willingham et al. [9] that equivalent contact time and interfacial area between two reactants for both arrays led to similar product formation; hence grain size alone cannot be an indicator to account for transverse mixing. In this work, the closely packed array has a lower porosity, more interfacial area and a smaller diffusion time-scale $\left(\tau_{D}=l^{2} / D_{C}\right)$ between the two reactants than the loosely packed array, resulting in more transverse mixing and product formation. It is also apparent that the effect of viscosity variation on the velocity field was more significant in the closely packing array resulting in more product formation.

Fig. 8 shows the concentration profiles in the cross flow direction along the outflow boundary of the domains for the same cases shown in Figs. 5 and 6, but also including the intermediate value of the reaction rate constant. In either array, the channeling for viscous thinning is reflected in the shape of the profiles, showing a peaked shape as the centerline is approached. The increased dispersion for viscous thickening is also evident on either array, with wider tails away from the centerline. For a particular array and viscosity type, transverse spreading is largely independent of $k$ value, but a higher $k$ value results in a higher magnitude of concentration near the centerline.

\subsection{Effect of viscosity variations on transverse dispersion}

Values of transverse dispersion coefficient $\left(D_{T, R}\right)$ were computed using product mass as a function of distance using Eq. (20). Although Eq. (20) is a continuum-scale analytical solution under uniform velocity condition, Eq. (20) has been successfully applied to compute apparent transverse dispersion coefficients for heterogeneous array patterns $[8,25]$. In contrast to previous studies, in this study the velocity field is affected by viscosity variations; thickening viscosity results in flow diversion away from the centerline and thinning viscosity results in flow focusing along the centerline. Asymptotic values of $D_{T, R}$ are plotted in Fig. 9 as a function of the Damkohler number $(D a)$. This plot shows more dispersion with thickening viscosity than with thinning viscosity, independent of $D a$ and array type. The case of constant viscosity falls in between the thickening and thinning cases (except at high $P e$ and $D a$, more below). The $D_{T, R}$ values were higher in the closely packed array than in the loosely packed array, which results from the increased interfacial area and smaller diffusion time-scale between the reactants in the closely packed array. Thickening 


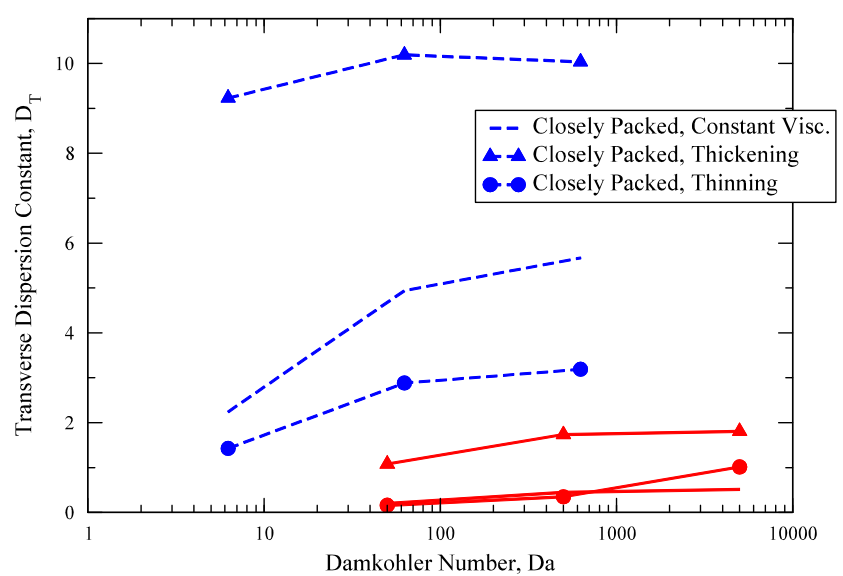

(a)

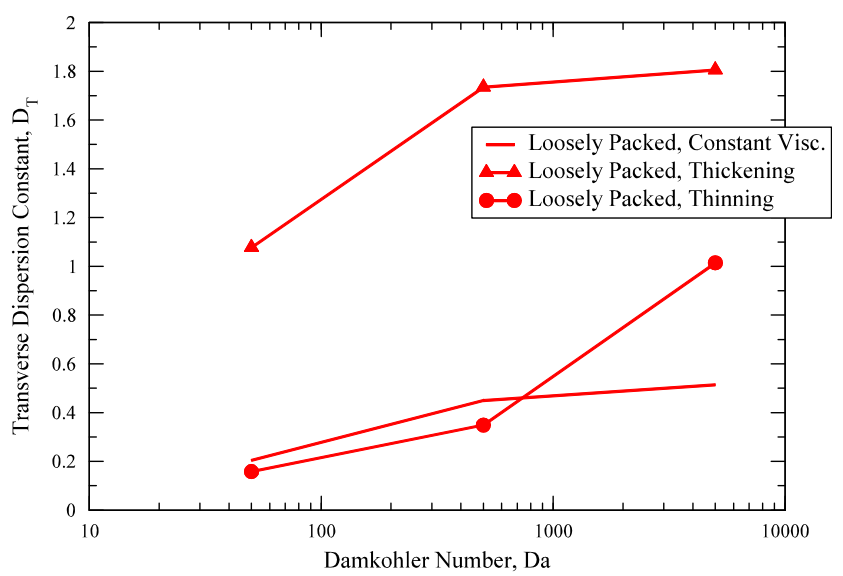

(b)

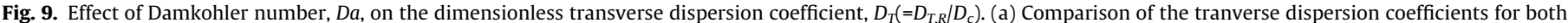
the loosely and closely packed cases. (b) Zoomed in view of the coefficients for the loosely packed case.

viscosity can enhance the transverse concentration gradient by altering reaction time scales as well as the flow field, resulting in higher $D_{T, R}$ values. The effect of flow focusing on mixing and product formation was experimentally and numerically investigated at the pore scale in Willingham et al. [25] and at the continuum scale in Rolle et al. [41]. The pore-scale study [25] shows that the dispersion coefficient was lower due to streamline compression in the focusing zone. Similarly, in this study, thinning viscosity results in lower $D_{T, R}$ values.

As shown in Fig. 8, the effect of reaction rate constant $k$ on total product mass over the outflow cross section was small for $k>1$. This is reflected in the calculated values of $D_{T, R}$ for both packing arrays which appear to be approaching an asymptotic value independent of $D a$ (for large $D a$ ). The one exception is the thinning viscosity case on the loosely packed array, which is associated with the instability discussed in Section 4.1. The instability and resultant transfer from one flow pattern to its opposite results in the movement of the reaction zone across the centerline of the channel. The movement of the reaction zone creates additional area for interaction of the reactants leading to the increased creation of product seen in Fig. 8 (b) and the higher value of $D_{T, R}$ in Fig. 9.

\section{Conclusions}

A model was developed for calculation of flow through porous media with chemical reaction dependent viscosity. Application of the model to two arrangements of porous media over a range of reaction rates indicates that viscosity changes can have a large effect on the production and distribution of product species. When the reaction products cause an increase in viscosity, the transverse distribution of product is larger than the constant viscosity case due to the flow field moving away from the centerline. The viscous thinning case results in the opposite situation where the increased fluid velocity near the centerline results in a reduced transverse distribution of product. The transverse dispersion is also increased in the closely packed array compared to the loosely packed array, due to the decreased pore spacing and corresponding increased interfacial contact area between the reactants in the closely spaced array. Aside from the viscosity behavior, the scaling shows the fundamental parameters to be the Damkohler number, Peclet number, and porosity.

An interesting result was the case of viscous thinning at high reaction rate on the loosely packed array. Here an instability was present and resulted in higher product concentration compared to the constant viscosity case at the same conditions. The moving instability increased the interaction between the two reactants resulting in the higher product concentration. This result motivates future study at higher $P e$ and $D a$ numbers to determine if other instabilities are present under a broader set of conditions. Higher fluid velocities generally result in a reduction of product formation due to reduced reaction time, but exploiting instabilities would allow for use of higher flow throughputs, while maintaining product formation rates.

Additionally, our pore scale modeling results suggest that the newly-developed pore scale model can be used to examine engineered fluids with adaptable properties including density. An example may be fluids whose viscosity depends on the local shear rate instead of the reaction products. Shear thinning fluids have use in enhanced oil recovery $[42,43]$ and subsurface remediations $[40,44]$. An alternative future path would be to include more realistic and complicated chemical reactions. A chemical reaction where the product results in precipitation or dissolution of a solid phase produces local change in the fluid viscosity. Applications include engineered fluids $[45,46]$ and natural reactions in relation to subsurface $\mathrm{CO}_{2}$ sequestration.

\section{Acknowledgements}

H.Y. and M.J.M. were supported as part of the Center for Frontiers of Subsurface Energy Security, an Energy Frontier Research Center funded by the US Department of Energy, Office of Science, Office of Basic Energy Sciences under Award Number DESC0001114. S.M.D. was supported by the Sandia National Laboratories Laboratory Directed Research and Development program. We also acknowledge the effort of three anonymous reviewers for their careful and constructive reviews, which led to improvement of our manuscript.

Sandia National Laboratories is a multi-program laboratory operated by Sandia Corporation, a wholly owned subsidiary of Lockheed Martin Company for the United States Department of Energy's National Nuclear Security Administration under contract DE-AC04-94AL85000.

\section{References}

[1] Cirpka OA, Frind EO, Helmig R. Numerical simulation of biodegradation controlled by transverse mixing. J Contam Hydrol 1999;40(2):159-82. 
[2] Knutson C, Werth CJ, Valocchi AJ. Pore scale simulations of biomass growth along the transverse mixing zone of a model 2D porous medium. Water Resour Res 2005;41:W07007. doi:10.1029/2004WR003459.

[3] Johnson JW, Nitao JJ, Knauss KG. Reactive transport modeling of $\mathrm{CO}_{2}$ storage in saline aquifers to elucidate fundamental processes, trapping mechanisms, and sequestration partitioning. In: Baines SJ, Worden RH, editors. Geological storage of carbon dioxide. London: Geological Society of London; 2004. p. 107-28.

[4] Zhang C, Dehoff K, Hess N, Oostrom M, Wietsma TW, Valocchi AJ, et al. Porescale study of transverse mixing induced $\mathrm{CaCO}_{3}$ precipitation and permeability reduction in a model subsurface sedimentary system. Environ Sci Technol 2010;44(20):7833-8.

[5] Homsy GM. Viscous fingering in porous media. Ann Rev Fluid Mech 1987;19:271-311.

[6] Pojman JA, Gunn G, Patterson C, Owens J, Simmons C. Frontal dispersion polymerization. J Phys Chem B 1998;102:3927-9.

[7] Knutson C, Valocchi AJ, Werth CJ. Comparison of continuum and pore-scale models of nutrient biodegradation under transverse mixing conditions. Adv Water Resour 2007;30(6-7):1421-31.

[8] Acharya RC, Valocchi AJ, Werth CJ, Willingham TW. Pore-scale simulation of dispersion and reaction along a transverse mixing zone in two-dimensional porous media. Water Resour Res 2007;43:W10435. doi:10.1029/ 2007WR005969.

[9] Willingham TW, Werth CJ, Valocchi AJ. Evaluation of the effects of porous media structure on mixing-controlled reactions using pore-scale modeling and micromodel experiments. Environ Sci Technol 2008;42(9):3185-93.

[10] DeWit A, Homsy GM. Viscous fingering in reaction-diffusion systems. J Chem Phys 1999;110:8663-75.

[11] DeWit A, Homsy GM. Nonlinear interaction of chemical reactions and viscous fingering in porous media. Phys Fluids 1999;11:949-51.

[12] Saffman PG, Taylor GI. The penetration of a fluid into a porous medium or Hele-Shaw cell containing a more viscous liquid. Proc R Soc Lond A 1958;245:312-29.

[13] Tanveer S. Surprises in viscous fingering. J Fluid Mech 2000;409:273-308.

[14] Christie MA. High-resolution simulations of unstable flows in porous media. SPE Reserv Eng 1989;4:297-304.

[15] Blunt MJ, Barker JW, Rubin B, Mansfield M, Culverwell ID, Christie MA. Predictive theory for viscous fingering in compositional displacement. SPE Reserv Eng 1994;9:73-80.

[16] Garcia JE, Pruess K. Flow instabilities during injection of $\mathrm{CO} 2$ into saline aquifers. In: Proceedings of TOUGH symposium, Lawrence Berkeley National Laboratory, Berkeley, California; 2003.

[17] Bandara U, Tartakovsky AM, Palmer B. Pore-scale study of capillary trapping mechanism during $\mathrm{CO}_{2}$ injection in geological formations. Int J Greenhouse Gas Control 2011;5:1566-77. doi:10.1016/j.ijggc.2011.08.014.

[18] Nagatsu Y, Matsuda K, Kato Y, Tada Y. Experimental study on miscible viscous fingering involving viscosity changes induced by variations in chemical species concentrations due to chemical reactions. J Fluid Mech 2007;571:475-93.

[19] Nagatsu Y, Kondo Y, Kato Y, Tada Y. Effects of moderate Damköhler number on miscible viscous fingering involving viscosity decrease due to a chemical reaction. J Fluid Mech 2009;625:97-124.

[20] Nagatsu Y, Kondo Y, Kato Y, Tada Y. Miscible viscous fingering involving viscosity increase by a chemical reaction with moderate Damköhler number. Phys Fluids 2011;23. 014109-1 - 8.

[21] Gérard T, De Wit A. Miscible viscous fingering induced by a simple $A+B \rightarrow C$ chemical reaction. Phys Rev E 2009;79. 016308-1 - 10.

[22] Raje D, Kapoor V. Experimental study of bimolecular reaction kinetics in porous media. Environ Sci Technol 2000;34(7):1234-9.

[23] Tartakovsky AM, Tartakovsky DM, Meakin p. Stochastic Langevin model for flow and transport in porous media. Phys Rev Lett 2008;101:044502. doi:10.1103/PhysRevLett.101.044502.
[24] Tartakovsky AM. Langevin model for reactive transport in porous media. Phys Rev E 2010;82:026302. doi:10.1103/PhysRevE.82.026302.

[25] Willingham TW, Zhang C, Werth CJ, Valocchi AJ, Oostrom M, Wiestma TW Using dispersivity values to quantify the effects of pore-scale flow focusing on enhanced reaction along transverse mixing zone. Adv Water Res 2010;33:525-35.

[26] Martinez MJ, Stone CM, Notz PK, Turner DZ, Hopkins PL, Subia S, et al Computational thermal, chemical, fluid, and solid mechanics for geosystems management. Technical Report, SAND2011-6643, Sandia National Laboratories, Albuquerque, NM 2011.

[27] Notz PK, Subia SR, Hopkins MM, Moffat HK, Noble D. Aria 1.5: User Manual Technical Report, SAND2007-2734, Sandia National Laboratories, Albuquerque, NM; 2007.

[28] Sangani AS, Acrivos A. Slow flow past periodic arrays of cylinders with application to heat transfer. Int J Multiphase Flow 1982;8(3):193-206.

[29] Nambi IM, Werth CJ, Sanford RA, Valocchi AJ. Pore-scale analysis of anaerobic halorespiring bacterial growth along the transverse mixing zone of an etched silicon pore network. Environ Sci Technol 2003;37(24):5617-24.

[30] Brezzi F. Existense, uniqueness, and approximation of saddle-point problems arising from Lagrange multipliers. Rev Fr Automat Infor 1974;8(NR2):129-51.

[31] Dohrmann CR, Bochev PB. A stabilized finite element method for the Stokes problem based on polynomial pressure projections. Int J Numer Methods Fluids 2004;46:183-201.

[32] Bochev PB, Dohrmann CR, Gunzburger MD. Stabilization of low-order mixed finite elements for the Stokes equations. SIAM J Numer Anal 2006;44(1):82-101.

[33] Gartling DK, Dohrmann CR. Quadratic finite elements and incompressible viscous flows. Comput Methods Appl Mech Eng 2006;195:1692-708.

[34] Christon MA, Martinez MJ, Voth TE. Generalized Fourier analysis of the advection-diffusion equation - Part I: One-dimensional domains. Int J Numer Methods Fluids 2004;45:839-87.

[35] Martinez MJ. Comparison of Galerkin and control volume finite element for advection-diffusion problems. Int J Numer Methods Fluids 2006;50:347-76.

[36] Gebart BR. Permeability of unidirectional reinforcements for RTM. J Compos Mater 1992;26:1100-33.

[37] Yazdchi K, Srivastava S, Luding S. Microstructural effects on the permeability of periodic fibrous porous media. Int J Multiphas Flow 2011;37(8):956-66.

[38] Feeney R, Schmidt SL, Strickholm P, Chadam J, Ortoleva P. Periodic precipitation and coarsening waves - applications of the competitive particle growth-model. J Chem Phys 1983;78(3):1293-311.

[39] Yoon H, Valocchi AJ, Werth CJ, Dewers T. Pore-scale simulation of mixinginduced calcium carbonate precipitation and dissolution in a microfluidic pore network. Water Resour Res 2011; Submitted for publication.

[40] Zhong L, Oostrom M, Wietsma TW, Covert MA. Enhanced remedial amendment delivery through fluid viscosity modifications: experiments and numerical simulations. J Contam Hydrol 2008;101:29-41.

[41] Rolle M, Eberhardt C, Chiogna G, Cirpka OA, Grathwohl P. Enhancement of dilution and transverse reactive mixing in porous media: experiments and model-based interpretation. J Contam Hydrol 2009;110:130-42.

[42] Johansen RT. Overview of selected oil recovery processes. J Rheol 1979;23:167-79.

[43] Lopez X, Valvatne PH, Blunt MJ. Predictive network modeling of single-phase non-Newtonian flow in porous media. J Colloid Interface Sci 2003;263:256-65.

[44] Martel KE, Martel R, Lefebvre R, Gelinas PJ. Laboratory study of polyme solutions used for mobility control during in situ NAPL recovery. Ground Water Monit R 1998;18(3):103-13.

[45] Espinosa D, Caldelas F, Johnston K, Bryant SL, Huh C. Nanoparticle-stabilized supercritical $\mathrm{CO}_{2}$ foams for potential mobility control applications. In: Proceedings of the 2010 SPE improved oil recovery symposium, Tulsa, Oklahoma; 2010, SPE paper 129925.

[46] Zhang T, Davidson A, Bryant SL, Huh C. Nanoparticle-stabilized emulsions for applications in enhanced oil recovery. In: Proceedings of the 2010 SPE improved oil recovery symposium, Tulsa, Oklahoma; 2010, SPE paper 129885. 